\title{
METAGENOMICS AND ITS RELEVANCE TO ANIMAL DISEASES AND GUT HEALTH
}

\author{
A. P. KOlTE ${ }^{1,2, *}$ A. Dhali ${ }^{1}$, P. K. MALiK ${ }^{1}$, D. T. PAL ${ }^{1}$ AND R. BhatTA ${ }^{1}$ \\ ${ }^{1}$ Omics Laboratory, ICAR-National Institute of Animal Nutrition and Physiology, Adugodi, \\ Bengaluru-560 030, Karnataka, India \\ ${ }^{2}$ Department of Biotechnology, Centre for Post Graduate Studies, Jain University, Bengaluru- 560 \\ 041, Karnataka, India
}

\begin{abstract}
Metagenomics offers exciting opportunity for diagnosis of infectious diseases. Although, it has a long way to go to find its place in diagnostic laboratories, several researchers have already proved its usefulness in diagnosis of difficult to culture pathogens. Simultaneous and unbiased detection of the microbiological agents is unique hallmark of these methods. The sequence data generated from samples can be used for identification of different classes of microbes, antibiotic resistance genes, mutations in the genes, even reconstructing genomes of the small organisms specially viruses. The two main approaches of metagenomic investigation are phylogenetic marker amplification popularly known as amplicon sequencing and shotgun metagenomics, which implies sequencing the total metagenomic DNA isolated from the samples. Metagenomics applied to the vectors offers possibility to screen all the microbes carried by the vectors and could be an important tool for epidemiological investigations. The review focuses on the approaches and applications of metagenomics, its usefulness and prospects.
\end{abstract}

Key words: Disease diagnosis, Metagenomics, NGS, Untargeted metagenomics, 16S rRNA amplicon sequencing

\section{Introduction}

During last century, several techniques were developed for studying microbes, including physiology, genetics and epidemiology. The identification of the bacterial pathogens in clinical settings is largely dependent on the techniques developed in late eighties. Veterinary diagnostic bacteriology is still practicing physical detection and characterization of causative bacteria under the microscope using techniques developed by Hans Christian Gram and isolation of the bacteria in colonies on petri plates that was initiated by Robert Koch. The microbes have different and specific need for growth on solid media which increases the workflow and skill requirements in the diagnostic microbiology laboratory. Sometimes, the carbon sources in the culture media are not exactly similar to the requirements of the bacterial growth reflecting into success in isolating only limited bacterial members of the community (Nocker et al., 2007).

Pathogen identification based on the traditional approach using morphology, physiology, chemistry, and biochemical characterization generally requires 2 to 5 days. In addition, phenotypic methods fail to identify the 
microorganism up to the species or strain level (Bochner, 2009). In some situations, the pathogens are difficult to visualize under microscope or the organisms are refractory to known culturing methods. The indirect approaches were thus developed that are independent of the culturing techniques such as, identification of specific proteins and /or nucleic acids. Molecular biology techniquesbased assays reduced the pathogen identification time (Castro-Escarpulli et al., 2015). Although, these approaches target specific molecules for individual pathogens therefore, it is difficult to detect unsuspected pathogens in the samples and requires multiple assays. The first-generation sequencing technology, Sanger sequencing, along with PCR based tests facilitated molecular identification and characterization of the pathogens and greatly aided in understanding of molecular epidemiology and host pathogen interactions. However, these technologies require prior knowledge of the pathogens and genomic sequences for clonal amplification. Beginning of the $21^{\text {st }}$ century has witnessed the strengths of nucleic acid-based tests to identify, characterize and strain type the microbes. Several PCR based tests were developed in past and have been successful in demonstrating the capabilities of identifying the microbial pathogens (Costa et al., 2014).

In case of bacterial pathogens, the discrepancy between the cultured diversity and in situ existing diversity resulted in adoption of the culture independent techniques for study of the bacterial communities in different niches (Hugenholtz et al., 1998; Zoetendal et al., 2004). The next generation sequencing techniques have an advantage that they are capable of identifying large number of pathogens simultaneously. The advancements in the Sanger chemistry based nucleic acid sequencing technologies have brought the sequencing-based tests in the diagnostic laboratories. The 16S rRNA gene was being used in identification of the microbes. The $16 \mathrm{~S}$ rRNA based phylogeny approach was introduced by Carl Woese in 1987 with 12 bacterial phyla with a few culturable representatives in each. Despite the 16S rRNA based lineages not being officially recognized (due to the continuous discovery of sequence signatures belonging to undescribed phyla), presently, the ARB-Silva database lists 67 phyla, including 37 candidate phyla; the Ribosomal Database Project 10, lists 49 phyla, including 20 candidate phyla; and National Centre for Biotechnology Information (NCBI) lists 120 phyla, including 90 candidate phyla. The phyla other than the candidate phyla do not have cultured representatives.

\section{Metagenomics}

It was a common belief that the organisms easily cultured from an ecosystem are numerically and functionally significant ones. However, later it was proven that these organisms are rarely dominant (Hugenholtz, 2002). These organisms usually get isolated due to their ability to grow in nutrient rich media at moderate temperature and under routine laboratory conditions. The cultivable organism's proportion constitutes less than 1$10 \%$ of the total microbial diversity (Prakash et al., 2013). The fact was earlier known as "the great plate count anomaly" but the unculturability could not be proven till the advent of molecular biology tools. Sequencing of the phylogenic marker genes was introduced to identify uncultured microbes in the environment. This approach was used largely to reconstruct phylogenies, comparison of microbial distribution in samples employing sequencing or restriction fragnent lenth polymorphism (RFLP) and quantification of relative abundance of each taxonomic group using hybridization with group-specific probes and primers. 
Metagenomics helps to identify the diversity, to study the population structure and to screen and isolate genes of our interest from the members which are yet to be cultured. The $16 \mathrm{~S}$ rRNA based phylogeny has paved way for faster identification of the pathogens as well as differentiation among the closely associated species based on the full length 16S rRNA gene sequence. The technique was developed for identification of yet to be cultured microbes from the environmental niches. The word 'metagenome' was first used by Handelsman et al. (1998) and refers to sequence-based study of collection of all microbial genomes found in a sample. Later, the technique was used extensively for identifying unculturable microbes from different ecosystems as well as directly identification of the functional enzymes from the metagenomic samples. The 16S rRNA based phylogeny, in combination with metagenomics approach, has allowed unbiased comparisons of microbial community members across various biological niche areas. A similar marker gene, $18 \mathrm{~S}$ rRNA can be used for identification of eukaryotic microbes like unicellular and multicellular parasites. Although, this approach has limitations in resolving the microbial diversity lower taxonomic ranks and the universal primers are not truly universal and does not guarantee the representation of all the microbes in the sample. This approach can identify the species of the microbe but fails to provide information on the subspecies or strain information that is vital for the diagnosis in terms of pathogenicity or antibiotic resistance. This shortcoming has limited the use 16S rRNA based metagenomics into the diagnosis of microbial diseases. A better resolution can be achieved using shotgun metagenomics approach. The term 'shotgun metagenomics' is used to define a methodology of direct sequencing of DNA extracted from a sample without culture or enrichment. This approach is used for clinical samples in the hope of detecting and characterizing pathogens.

\section{Metagenomic approaches}

Sequencing the samples for metagenomic diagnosis has two main approaches. The first approach uses amplification of the phylogenic marker genes (preferably the 16/18S rRNA gene) after PCR amplification. The universal presence of the selected phylogenic gene in the organisms facilitates simultaneous detection of several organisms in the sample. The approach is popularly known as amplicon sequencing and a typical workflow is given in Fig. 1. Amplicon sequencing can be used for the samples where the input sample contains tissue or sample matrix that can contribute to the metagenomic DNA. Sample multiplexing; running several samples in a single sequencing run on the next generation sequencer has further reduced the costs and affordability of the technology.

The 16S rRNA gene contains 9 hypervariable regions spanned with constant regions. Parts of the 16S rRNA genes that are not under strong negative selection, the mutations tend to accumulate, and variable regions are formed within the gene. The variable/hypervariable regions are flanked by the conserved regions that constituted the basis of designing the universal primers for simultaneous amplification of variable regions for microbial phylogeny. The amplified 16S rRNA genes from the samples are sequenced using next generation sequencing technologies and the sequence data is used for identification of the microbial composition within the samples. Initially, the term 'next-generation sequencing' was used to describe the high-throughput sequencing chemistry from classical Sanger sequencing. Later, they were recognized as second generation sequencing technologies that were based on nano engineered platforms facilitating the simultaneous sequencing of millions of nucleic acid molecules in one setup and capable of generating several gigabases $(\mathrm{Gb})$ of sequence data that could be used for genome sequencing, variant detections, gene activities and basic understanding of the host pathogen interactions. 


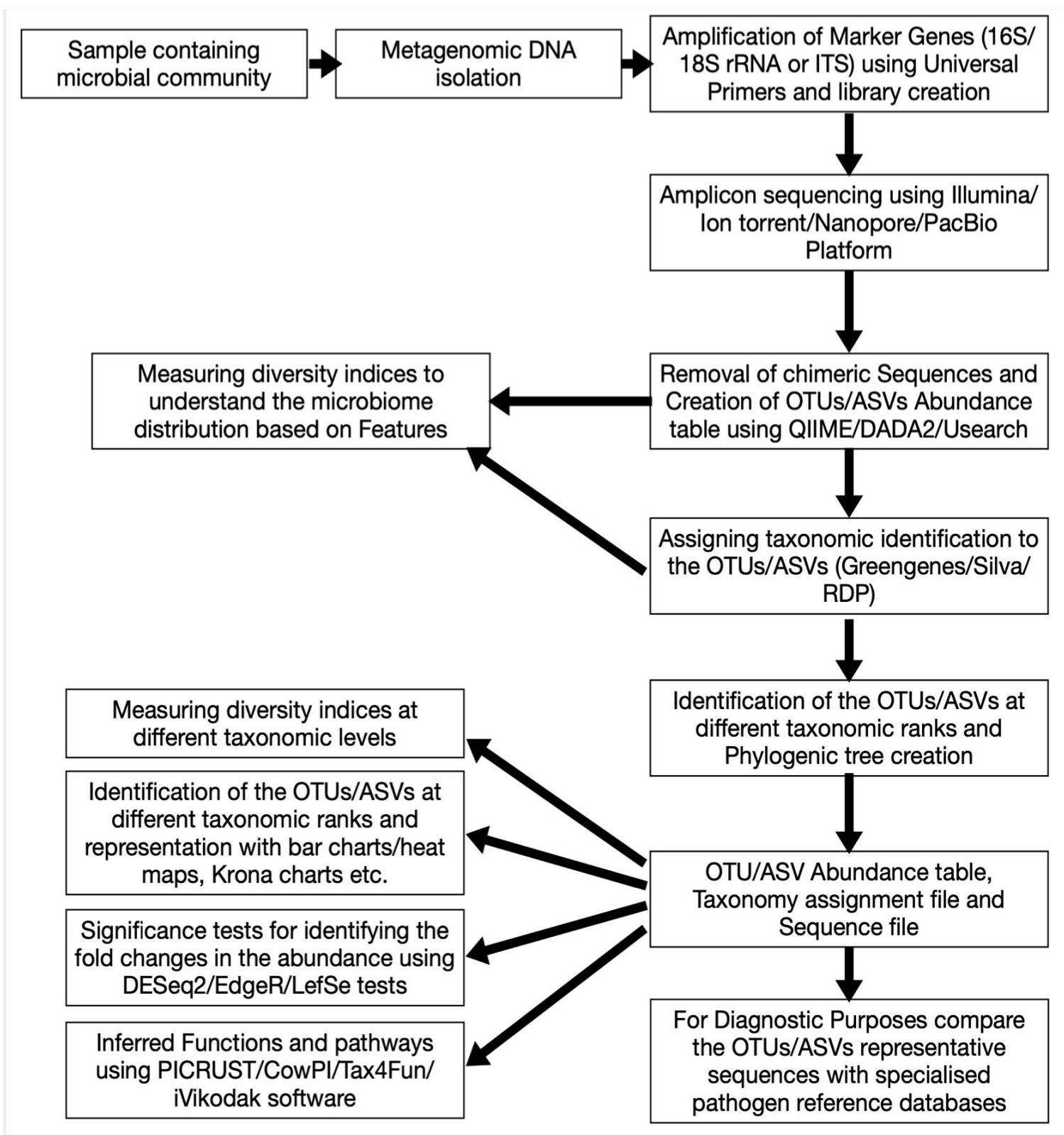

Fig. 1. A typical workflow of targeted amplicon sequencing and bioinformatic analysis

However, the approach could not generate the full length $16 \mathrm{~S}$ rRNA gene. The secondgeneration technologies needed clonal amplification of the input DNA molecules to produce detectable signal for sequencing. The third-generation sequencing technologies rely on directly sequencing the nucleic acid molecules directly. The third-generation sequencing technology is still in infancy and suffers from the throughput requirements of open-ended diagnostic utility. Currently, NGS techniques are classified as second- and third-generation sequencing methods.

The second-generation technologies have limitations to sequence the complete $16 / 18 \mathrm{~S}$ rRNA genes, which is crucial for taxonomic identification of the microbes up to species and strain level. Further, this approach excludes the viral counterpart which is one of the most 
important pathogen classes from animal health as well as zoonotic point of view. Shotgun metagenomics was successfully used to identify all types of microbes from the sequence data (Pallen, 2014). The term shotgun metagenomics refers to sequencing of the total DNA isolated from the sample and is done by using k-mer based taxonomic classifier software. A typical shotgun metagenomics workflow is presented in Fig. 2.

\section{Metagenome and gut health}

The largest portion of the host associated microbes are present in the gut of the monogastric animals and in the rumen in case of ruminant animals. The microbes perform various metabolic, physiological and immunological functions (Malmuthuge and Gua, 2016). By virtue of the diverse functional contribution of the gut microbiome, it has been recognized as an organ of the body. Since the initial colonization immediately after birth these microbes are involved in various functions in rumen as well as intestine (Fig. 3). Recently, the rumen microbes are shown to have association with important economic traits in livestock like feed efficiency (Berry and Crowley, 2012; Shabat et al., 2016; Delgado et al., 2019; Li et al., 2019), enteric methane production (Wallace et al., 2015; Roehe et al.,

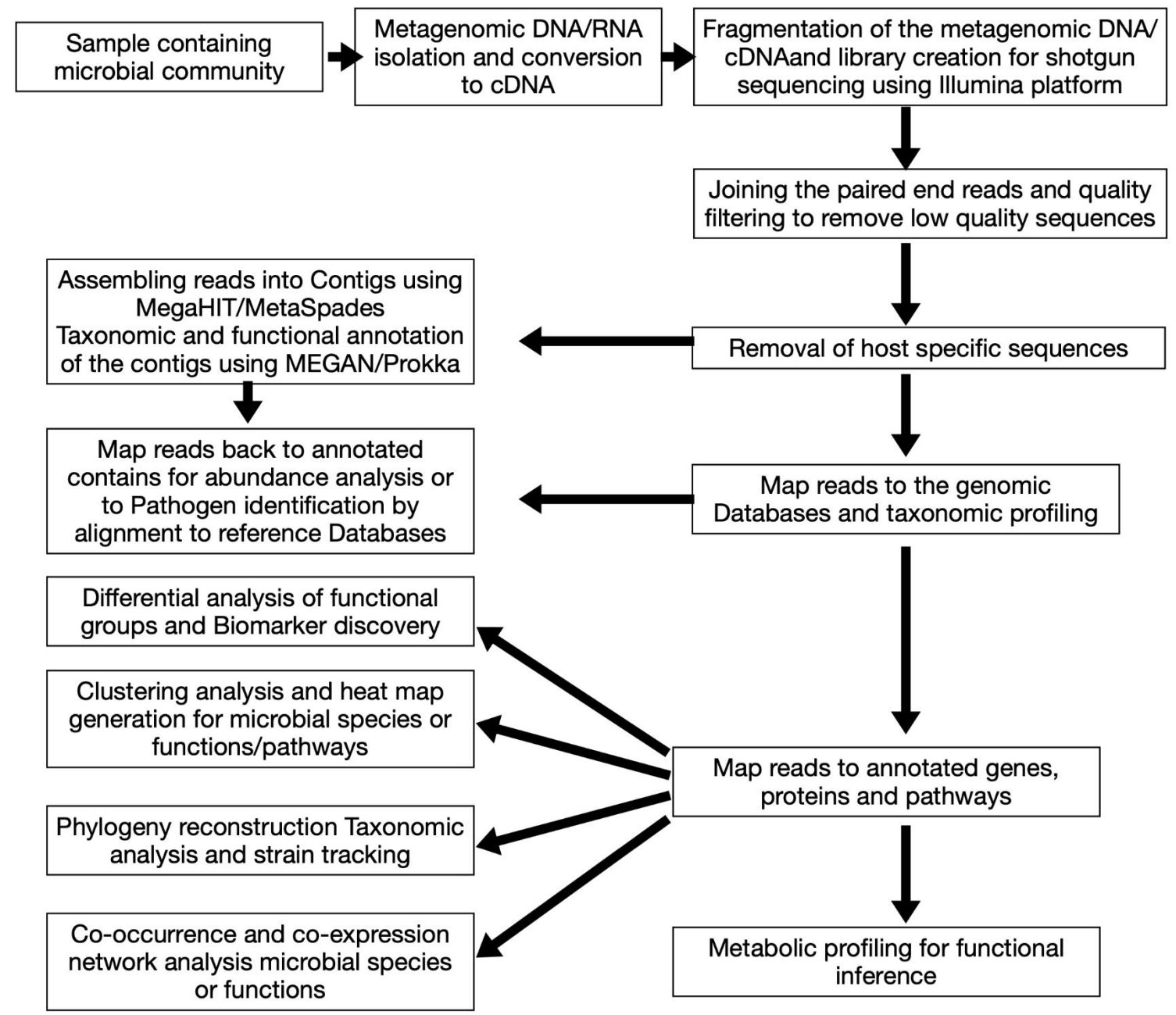

Fig. 2. Typical workflow of shotgun metagenomics used for microbial profiling and disease investigation 


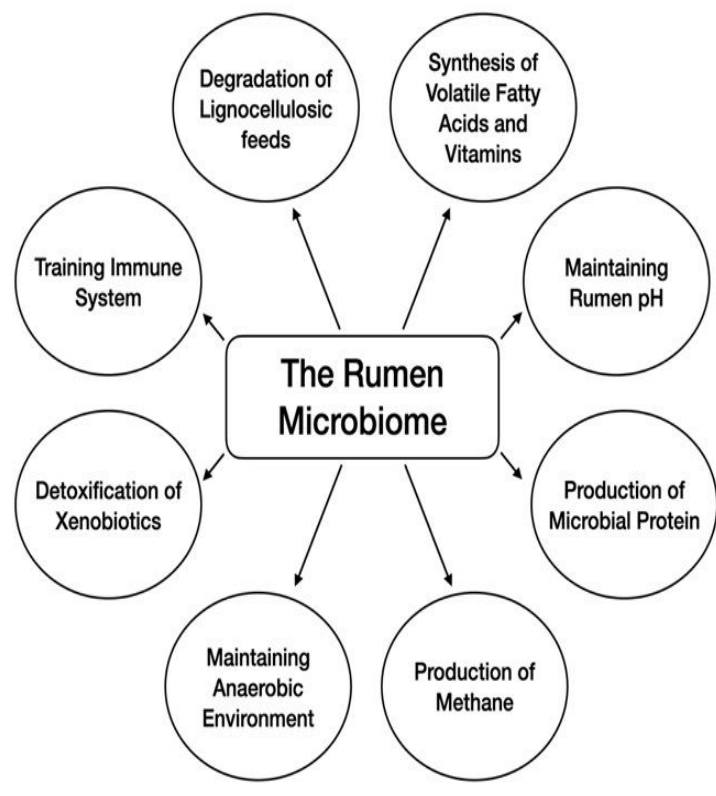

(a)

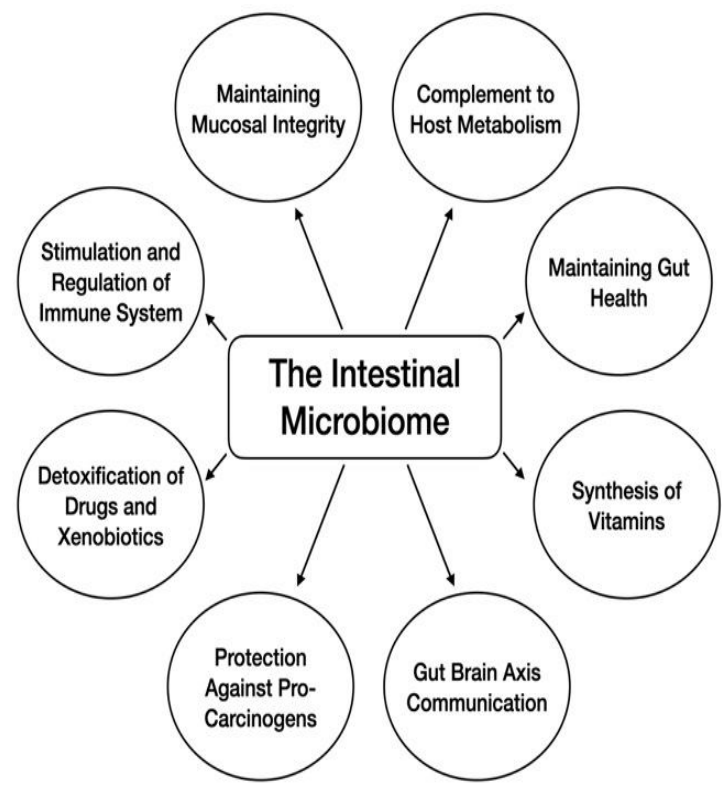

(b)

Fig. 3. Functions of rumen microbiome (a) and intestinal microbiome (b) in ruminants

2016; Difford et al., 2018), milk composition (Jami et al., 2014; Xue et al., 2019) and ruminal acidosis (McCann et al., 2016). Ruminal acidosis causes dysbiosis in the microbiome composition and ruminal fluid transplantation from healthy counterpart has shown recovery in sheep (Liu et al., 2019). The lower tract microbiome is suggested to be involved in maintaining the animal health and interaction with the immune system (Lyte et al., 2018).

\section{Limitations of metagenomic diagnosis}

Although, metagenomics promises unprecedented opportunity to detect the pathogens in the samples of human, animal and food source, the success of metagenomic exploration depends upon several factors. The first requirement is isolation of the nucleic acids from the samples for sequencing. As the samples are from different composition, the methods for isolation of the nucleic acids are different depending on the sample matrix and contaminating substances. Several laboratories are standardizing methodologies for isolation of high-quality nucleic acids from different clinical samples. Additionally, the data generation requirements are dependent on the amount of input nucleic acids present in the samples. Lower the template higher data needs to be generated from the sequencing platform. The data handling capabilities demand computing infrastructure and specialized software. Expertise in the bioinformatics is required for analysis of the data. And finally, the reference pathogen databases for the investigation are required. Presently the databases and analysis pipelines are being developed at several laboratories, but due to dynamic nature of the databases, the pipelines are also constantly updating, and one need to keep pace with the newer methods continuously.

The targeted amplicon sequencing, being simple and less time consuming, has following advantages. It can be used for identification of multiple pathogens simultaneously in a single test. It targets the specific microbial groups 
viz.16S rRNA for bacteria and archaea (Rampini et al., 2011; Salipante et al., 2013), 18S rRNA for eukaryotes, ITS regions for fungi (Wagner et al., 2018) and RNA dependent RNA Polymerase (RdRP) for RNA viruses (Culley et al., 2010). It generates relative abundance of the microbial groups in the sample therefore changes in overall microbiome composition can be studied. The technique can capture rare taxa if the depth of sequencing is appropriate. Taxonomical classification is easier, and the reference databases are smaller that can be handled on desktop computers. Curated databases are available for comparison. The approach is suitable for low biomass samples like body fluids as the nucleic acid content is usually low for whole metagenome sequencing. The cost involvement is lower as compared to the whole metagenome sequencing. However, the method has certain disadvantages. It still requires prior knowledge of the microbial group targets and databases availability. If the organism is not present in the reference database used, the potential novel organisms can go undetected. Universal targets sometimes are not truly universal (i.e. the gene may be present, but the chosen primers may not amplify due to mismatches). As the technique requires amplification step, PCR bias can affect the outcome, which could influence the compositional comparisons across the samples. Due to the PCR bias, absolute abundances cannot be estimated correctly. As the secondgeneration sequencing platforms sequence maximum 500-550 base pairs, it does not cover the full-length marker gene; therefore the identification up to species and further levels cannot be accurately done. Although, the problem can be solved by third generation sequencing technologies, but at present the cost involvement is prohibitively high. The use of larger datasets for comparisons requires computational infrastructure, dedicated data processing pipelines and bioinformatics expertise.
The shotgun metagenomics, also regarded as unbiased approach to estimate and identify the microbial diversity, is better on the following terms. The technique can sequence all the DNA in the sample including bacteria, archaea, viruses and eukaryotes including the parasites and vectors. It is possible to filter the universal marker data from the shotgun metagenomics for using taxonomic identifications. The approach generates total genomic data; therefore, the functional aspects of the microbiomes can be interpreted that can provide opportunity for identifying the virulence factors, antibiotic resistance genes, antimicrobial proteins, functional pathways etc. Further, for both the approaches, online portals are available where data can be uploaded, and results can be visualized or downloaded. Several data analysis pipelines have been developed for handling the clinical metagenome data (Mulcahy-O'Grady and Workentine, 2016). However, as the data generated by shotgun metagenomics is large, in gigabases, it requires more computational power. The assembly of the metagenome is required before using the data for taxonomic or functional assignments. Particularly forviromes, the sample needs enrichment or exclusion of the other microbes that can contribute to the sequence data. High abundance of the host DNA and taxonomically uninformative sequences sometimes present in the data which does not contribute to the outcome and need to be removed using bioinformatics software. Costs are higher as more depth of sequencing is required for complex samples and particularly if the organisms of interest are in low abundance. For example, to detect 10 cells/g sample, probably the metagenomic data needs to be generated to the tune of approximately 400 Giga base pairs (Suttner et al., 2020). These requirements can be further depending on the overall complexity of the samples. High bioinformatics technical expertise or human resource is required for data 
handling and interpretation as well as large datasets needs a plenty of digital data storage space.

\section{Metagenomics in diagnosis}

In situations, where the conventional and molecular tests-based diagnostics fail to identify the causative agents in the samples, the metagenomic approach that is basically culture free and faster, might provide an answer. Today, it is increasingly evident that the differences in host-associated microbial communities can influence the balance between health and disease in conditions not normally thought of as microbial or infectious in origin: for example, inflammatory bowel disease, cancer or obesity. Sometimes, it may not be sufficient to focus diagnostic efforts on single pathogen in clinical samples that is thought to cause disease. Instead, it is now recognized that interactions between organisms in a community can influence disease outcome and, in some cases, it might even be appropriate to treat a whole microbial community as a pathogenic entity. Given the difficulty of culturing most of the viruses, shotgun sequencing to identify and detect human-associated viruses has been tried. The genomes of DNA viruses can be recovered through shotgun sequencing of DNA directly extracted from a sample. To detect RNA viruses, RNA extracted from a sample has to be converted to cDNA (Batty et al., 2013). The first use of metagenomics for presumptive diagnosis was reported by Wilson et al. (2014) for diagnosis of leptospirosis in the cerebrospinal fluid (CSF) by amplicon sequencing that was not detected in a control sample. The diagnosis was further confirmed by specific molecular tools and serology.

In a first study, fecal metagenomics was used to detect bacterial pathogens (Nakamura et al., 2008). 156 Campylobacter sequences were found in a sample taken during a bout of illness but were absent from a convalescent sample from the same individual. The potential of diagnostic metagenomics was demonstrated on stool samples collected during the outbreak of Shiga-toxigenic E. coli O104:H4 in Germany during May-June 2011 (Loman et al., 2013). The authors could get deep coverage of the outbreak strain genome from several stool metagenomes, Illumina MiSeq bench top sequencer and subsequently using higherthroughput instrument, HiSeq2500, also recovered genome-level coverage of other pathogens like Campylobacter jejuni, Clostridium difficile, Salmonella enterica, that had been detected by routine microbiological investigation in several STEC-negative samples. This study clearly established the proof-ofprinciple that metagenomics could be used not only to detect, but also to characterize bacterial pathogens within a sample. Next generation sequencing of the pig saliva samples revealed that Streptococcus was most abundant genera and $S$. suis was the most abundant species suggesting that the pig saliva is a potent source of $S$. suis infection to piglets and animal handlers (Murase et al., 2019). In another study metagenomics was used to identify $S$. suis, a zoonotic pathogen, in a patient whose blood bacterial cultures were negative to post antibiotic therapy (Dai et al., 2019). Culturenegative sample of necrotic hepatitis using whole-metagenome shotgun sequencing was used to detect B. melitensis (Lazarevic et al., 2018). As Chlamydiae require labour-intensive culturing, the metagenomic method was used for characterizing the chlamydial plasmids in samples and a novel species of chlamydia was also reported (Taylor-Brown et al., 2017).

Pyrosequencing was used for identifying organisms associated with mastitis, a multi etiological syndrome. The mastitis milk samples were characterized by culture as Trueperella pyogenes and Streptococcus spp. The mastitis pathogens identified by culture were generally among the most frequent organisms detected 
by pyrosequencing. Further, in aerobic culturenegative samples, pyrosequencing identified bacterial pathogens for mastitis, those are known pathogens but so far not been associated with mastitis, and those are currently not known to be pathogens. A possible role of anaerobic pathogens in bovine mastitis is also suggested based on the study (Oikonomou et al., 2012). $16 \mathrm{~S}$ based phylogenomics was also demonstrated to evaluate a sample from various areas of a dairy farm for the presence of bacterial organisms associated with digital dermatitis lesions and successfully detected association of Treponema spp. with the lesions and the presence on the hoof trimming equipment (Rock et al., 2015). The whole metagenomic investigation of the multi etiologic diseases and syndromes may be useful in identifying the predisposing microbes or indicator microbes for the disease progression. Uterine microbiome profile in the metritis cows revealed shifts in the microbiome composition (dysbiosis), however the causative agents were present in low abundance (Jeon and Galvao, 2018). Metagenomic characterization of the bovine milk microflora from normal and clinical mastitis samples revealed presence of exclusive organisms in affected animals as well as human pathogens were also detected indicating poor hygiene of the milk production (Hoque et al., 2019). The same data was used for assessment of resistome profile in the clinical mastitis samples revealing presence of biofilm forming factors in pathogens, antibiotic/drug resistance genes, the information can be useful in selecting appropriate treatment schedule (Hoque et al., 2020).

Given the difficulty in culturing the viruses, diagnostic metagenomics can be a promising tool for viral disease diagnosis. Metagenomics was used in diagnosing fetal infections in transplant patients and identified sequences of lymphocytic choriomeningitis virus (Palacios et al., 2008). Several recent studies demonstrated the identification of viral agents in the ancient samples. A study of the Tyrolean ice mummy Ötzi genome revealed sequences of Borrelia burgdorferi, making it the first known case of Lyme disease (Keller et al., 2012). Krause and colleagues recovered Mycobacterium leprae genome from the metagenome obtained from a historical dental sample (Schuenemann et al., 2013). The first example of post-mortem metagenomic diagnosis of tuberculosis was done from mummified lung tissue of a young woman who died in 1797 (Chan et al., 2013). A novel species of Ebola virus (Bundibugyo ebolavirus) was also discovered using this approach from Uganda (Towner et al., 2008). In another study, a novel arenavirus responsible for a hospital outbreak of haemorrhagic fever in South Africa was identified (Briese et al., 2009).

Metagenomics was applied in veterinary science and diagnosis in recent past to detect several newly emerging or re-emerging diseases. The first virus of high emerging impact was identified by metagenomics is Schmallenberg virus (SBV), belonging to orthobunya virus of the Simbu serogroup (Hoffmann et al., 2012). The virus was identified from the blood samples of cattle in Netherlands and Germany showing drop in production and fever, though other diagnostic methods could not detect the cause. Later astroviruses were identified in cattle diagnosed with bovine encephalitis in Europe, influenza $\mathrm{D}$ virus in cattle in the United States and France, bat influenza viruses H17N10 and H18N11 in bats in Central and South America, and a novel zoonotic borna virus in variegated squirrels in Germany. From 2009 to 2017 almost 20 animal and zoonotic viral disease agents were diagnosed using metagenomic technique (Hoeper et al., 2017). A limited scale metagenomics-based pathogen surveillance study on 6 poultry farms detected the main infectious viruses of poultry, and it analyzed 
the subtypes, genotypes and pathogenicity of some detected viruses (Qiu et al., 2019). Subsequent to the metagenomic investigation of astroviruses in enteric disorders in humans (Finkbeiner et al., 2009), a divergent strain was demonstrated to be associated with the brain tissue of shaking mink syndrome affected minks (Blomström et al., 2010). Viral metagenomics can be a valuable tool for molecular epidemiological studies, it was successfully used to detect field rabies viruses from the data generated from the high throughput metagenome sequencing (Orlowska et al., 2019). Ticks are important vectors for different tick-borne viruses, viral metagenomics of the vector can be a promising tool to simultaneously identify all the viruses present in a sample, including novel variants of already known viruses or completely new viruses (Damian et al., 2020). An exhaustive common livestock species wise list of animal viruses detected using vial metagenomics approach is reviewed by Kwok et al. (2020).

Apart from the identification of the disease causative agents, the untargeted metagenomics can be used for identification of the antibiotic resistance (AMR) genes in the samples. Traditional AMR assessment is based on the phenotypic AMR assays, while metagenomic studies discover the genes responsible for AMR (Duarte et al., 2020). Recent evidence has indicated that the presence of AMR genes in isolates can be highly corelated with observed phenotypic resistance (Stubberfield et al., 2019; Guo et al., 2019).

\section{Present challenges to metagenomic approaches}

Although, metagenomics promises unprecedented opportunity to detect the pathogens in the samples of human, animal and food source, the success of metagenomic exploration depends upon several factors. The metagenomics in diagnosis has a long way to prove itself a validated tool. Several issues still need to be addressed like, sampling bias and optimum methods for nucleic acid extraction, estimation of required sequencing depth, affordable sequencing platforms, dedicated databases for faster diagnosis, and cost of diagnosis. The first requirement is isolation of the nucleic acids from the samples for sequencing. As the samples are from different composition, the methods for isolation of the nucleic acids are different depending on the sample matrix and contaminating substances. Several laboratories are standardizing methodologies for isolation of high-quality nucleic acids from different clinical samples. The viral RNA isolation from the metagenomic sample is challenging as the molecules are present in very low abundance in the total RNA of the sample and are highly labile. The RNA isolation protocols for each type of samples need to be standardized. Furthermore, the data generation requirements are dependent on the amount of input nucleic acids present in the samples. Lower the abundance of the pathogen in the sample, higher data needs to be generated from the sequencing platform. The data handling capabilities demand computing infrastructure and specialized software. Of course, developing capacity or human resource also needs to be addressed before bringing it to the diagnostic laboratories. Finally, the reference pathogen databases for the investigation are required. Presently the databases and analysis pipelines are being developed at several laboratories, but due to dynamic nature of the databases, the pipelines are also changing, and one need to keep pace with the newer methods continuously.

The prospects of metagenomics are lucrative as it can offer opportunity to detect unsuspected pathogens, simultaneous detection of multiple pathogens of different classes and taxonomic origin, as well as functional information of the pathogens. The 
untargeted approach basically does not depend on the prior knowledge of the pathogen and hence allows detection of different classes of pathogens in a single protocol (Nakamura et al., 2011). The tool can be valuable in zoonotic and epidemiological studies to understand the disease spread. It is already proven that the diagnostic metagenomics has already played a role in identifying the causes

\section{REFERENCES}

Batty EM, Wong TN, Trebes A, Argoud K, Attar M et al., 2013. A modified RNA-Seq approach for whole genome sequencing of RNA viruses from faecal and blood samples. PLoS One, 8(6): e66129, doi: 10.1371/journal.pone.0066129

Berry DP and Crowley JJ, 2012. Residual intake and body weight gain: A new measure of efficiency in growing cattle. J Anim Sci, 90(1): 109-115, doi: $10.2527 /$ jas.2011-4245

Blomström AL, Widén F, Hammer AS, Belák S and Berg M, 2010. Detection of a novel astrovirus in brain tissue of mink suffering from shaking mink syndrome by use of viral metagenomics. J Clin Microbiol, 48(12): 4392-4396, doi: 10.1128/JCM.01040-10

Bochner BR, 2009. Global phenotypic characterization of bacteria. FEMS Microbiol Rev, 33(1): 191205, doi: 10.1111/j.1574-6976.2008.00149.x

Briese T, Paweska JT, McMullan LK, Hutchison SK, Street C et al., 2009. Genetic detection and characterization of Lujo virus, a new hemorrhagic fever-associated arenavirus from southern Africa. PloS Pathog, 5(5): e1000455, doi: 10.1371/journal.ppat.1000455

Castro-Escarpulli G, Alonso-Aguilar NM, Rivera G, Bocanegra-Garcia V, Guo X et al., 2015. Identification and typing methods for the study of bacterial infections: A brief review and Mycobacterial as case of study. Arch Clin Microbiol, 7(1-3): 1-10

Chan JZ, Sergeant MJ, Lee OY, Minnikin DE, Besra GS et al., 2013. Metagenomic analysis of tuberculosis in a mummy. N Engl J Med, 369(3): 289-290, doi: 10.1056/NEJMc 1302295 of unknown illnesses and outbreaks. Although there are several challenges in fully implementing the technique for diagnosis, nonetheless, in a decade or two the technique will be method of choice for detecting the hard to culture microbes, unsuspecting agents and apart from the main causative agent, a tool to identify the associated opportunist pathogens in diagnostic microbiology.

Costa P, Botelho A, Couto I, Viveiros M and Inácio J, 2014. Standing of nucleic acid testing strategies in veterinary diagnosis laboratories to uncover Mycobacterium tuberculosis complex members. Front Mol Biosci, 1: 16, doi: 10.3389/ fmolb.2014. 00016

Culley AI, Suttle CA and Steward GF, 2010. Characterization of the diversity of marine RNA viruses. In: Wilhelm SW, Weinbauer MG, Suttle CA, (eds) Manual of Aquatic Viral Ecology. American Society of Limnology and Oceanography: Waco, TX, pp193-201

Dai Y, Chen L, Chang W, Lu H, Cui P et al., 2019. Culture-negative Streptococcus suis infection diagnosed by metagenomic next-generation sequencing. Front Public Health, 7: 379, doi: 10.3389/fpubh.2019.00379

Damian D, Maghembe R, Damas M, Wensman JJ and Berg M, 2020. Application of viral metagenomics for study of emerging and reemerging tick-borne viruses. Vector-Borne Zoonot Dis, 20(8): 557-565, doi: 10.1089/ vbz.2019.2579

Delgado B, Bach A, Guasch I, González C, Elcoso G et al., 2019. Whole rumen metagenome sequencing allows classifying and predicting feed efficiency and intake levels in cattle. Sci Rep, 9(1): 1-3, doi: 10.1038/s41598-01836673-w

Difford GF, Plichta DR, Løvendahl P, Lassen J, Noel SJ et al., 2018. Host genetics and the rumen microbiome jointly associate with methane emissions in dairy cows. PLoS Genet, 14(10): e 1007580, doi: 10.1371/journal.pgen. 1007580 
Duarte ASR, Stärk KD, Munk P, Leekitcharoenphon P, Bossers A et al., 2020. Addressing learning needs on the use of metagenomics in antimicrobial resistance surveillance. Front Public Health, 8: 38, doi: 10.3389/fpubh. 2020.00038

Finkbeiner SR, Li Y, Ruone S, Conrardy C, Gregoricus $\mathrm{N}$ et al., 2009. Identification of a Novel astrovirus (astrovirus VA1) associated with an outbreak of acute gastroenteritis. J Virol, 83(20): 10836-10839, doi: 10.1128/JVI.00998-09

Guo S, Tay MYF, Aung KT, Seow KLG, Ng LC et al., 2019. Phenotypic and genotypic characterization of antimicrobial resistant Escherichia coli isolated from ready-to-eat food in Singapore using disk diffusion, broth microdilution and whole genome sequencing methods. Food Control, 99: 89-97, doi: 10.1016/j.foodcont.2018.12.043

Handelsman J, Rondon MR, Brady SF, Clardy J and Goodman RM, 1998. Molecular biological access to the chemistry of unknown soil microbes: A new frontier for natural products. Chem Biol, 5(10): R245-R249, doi: 10.1016/ S1074-5521(98) 90108-9

Hoeper D, Wylezich C and Beer M, 2017. Chapter twoLoeffler 4.0: diagnostic metagenomics. Adv Virus Res, 99: 17-37, Doi: 10.1016/ bs.aivir.2017.08.001

Hoffmann B, Scheuch M, Höper D, Jungblut R, Holsteg $\mathrm{M}$ et al., 2012. Novel orthobunyavirus in cattle, Europe, 2011. Emerg Infect Dis, 18(3): 469-472, doi: $10.3201 /$ eid1803.111905

Hoque MN, Istiaq A, Clement RA, Gibson KM, Saha O et al., 2020. Insights into the resistome of bovine clinical mastitis microbiome, a key factor in disease complication. Front Microbiol, 11: 860, doi: $10.3389 /$ fmicb. 2020.00860

Hoque MN, Istiaq A, Clement RA, Sultana M, Crandall KA et al., 2019. Metagenomic deep sequencing reveals association of microbiome signature with functional biases in bovine mastitis. Sci Rep, 9: 13536, doi: 10.1038/s41598-019-49468-4

Hugenholtz P, 2002. Exploring prokaryotic diversity in the genomic era. Genome Biol, 3: 1-8

Hugenholtz P, Goebel BM and Pace NR, 1998. Impact of culture-independent studies on the emerging phylogenetic view of bacterial diversity. J Bacteriol, 180(18): 4765-4774, doi: 10.1128/ JB.180.18.4765-4774.1998

Jami E, White BA and Mizrahi I, 2014. Potential role of the bovine rumen microbiome in modulating milk composition and feed efficiency. PLoS One, 9: e85423, doi: 10.1371/journal.pone. 0085423

Jeon SJ and Galvão KN, 2018. An advanced understanding of uterine microbial ecology associated with metritis in dairy cows. Genomics Inform, 16(4): e21, doi: 10.5808/ GI.2018.16.4.e21

Keller A, Graefen A, Ball M, Matzas M, Boisguerin V et al., 2012. New insights into the Tyrolean Iceman's origin and phenotype as inferred by whole-genome sequencing. Nat Commun, 3(1): 1-9, doi: 10.1038/ncomms 1701

Kwok KTT, Nieuwenhuijse DF, Phan MVT and Koopmans MPG, 2020. Virus metagenomics in farm animals: A systematic review. Viruses, 12(1): 107, doi: 10.3390/v12010107

Lazarevic V, Gaïa N, Girard M, Leo S, Cherkaoui A et al., 2018. When bacterial culture fails, metagenomics can help: A case of chronic hepatic brucelloma assessed by next-generation sequencing. Front Microbiol, 9: 1566, doi: 10.3389/fmicb.2018.01566

Li F, Li C, Chen Y, Liu J, Zhang C et al., 2019. Host genetics influence the rumen microbiota and heritable rumen microbial features associate with feed efficiency in cattle. Microbiome, 7(1): 92, doi: 10.1186/s40168-019-0699-1

Liu J, Li H, Zhu W and Mao S, 2019. Dynamic changes in rumen fermentation and bacterial community following rumen fluid transplantation in a sheep model of rumen acidosis: implications for rumen health in ruminants. FASEB J, 33(7): 84538467, doi: 10.1096/fj.201802456R

Loman NJ, Constantinidou C, Christner M, Rohde H, Chan JZ et al., 2013. A culture-independent sequence-based metagenomics approach to the investigation of an outbreak of Shiga-toxigenic Escherichia coli O104: H4. JAMA, 309(14): 1502-1510, doi: 10.1001/jama.2013.3231 
Lyte M, Villageliú DN, Crooker BA and Brown DR, 2018. Symposium review: microbial endocrinology-why the integration of microbes, epithelial cells, and neurochemical signals in the digestive tract matters to ruminant health. J Dairy Sci, 101(6): 5619-5628, doi: 10.3168/ jds.2017-13589

Malmuthuge N and Guan LL, 2016. Gut microbiome and omics: a new definition to ruminant production and health. Anim Front, 6(2): 8-12, doi: 10.2527/af.2016-0017

McCann JC, Luan S, Cardoso FC, Derakhshani H, Khafipour E et al., 2016. Induction of subacute ruminal acidosis affects the ruminal microbiome and epithelium. Front Microbiol, 7: 701, doi: 10.3389/fmicb.2016.00701

Mulcahy-O'Grady $\mathrm{H}$ and Workentine ML, 2016. The challenges and potential of metagenomics in the clinic. Front Immunol, 7: 29, doi: 10.3389/ fimmu.2016.00029

Murase K, Watanabe T, Arai S, Kim H, Tohya M et al., 2019. Characterization of pig saliva as the major natural habitat of Streptococcus suis by analyzing oral, fecal, vaginal, and environmental microbiota. PloS One, 14(4): e0215983, doi: 10.1371/journal.pone. 0215983

Nakamura S, Maeda N, Miron IM, Yoh M, Izutsu K et al., 2008. Metagenomic diagnosis of bacterial infections. Emerg Infect Dis, 14(11): 1784-1786, doi: $10.3201 /$ eid1411.080589

Nakamura S, Nakaya T and Iida T, 2011. Metagenomic analysis of bacterial infections by means of high-throughput DNA sequencing. Exp Biol Med, 236(8): 968-71, doi: 10.1258/ ebm.2011.010378

Nocker A, Burr M and Camper AK, 2007. Genotypic microbial community profiling: A critical technical review. Microb Ecol, 54: 276-89, doi: 10.1007/s00248-006-9199-5

Oikonomou G, Machado VS, Santisteban C, Schukken YH and Bicalho RC, 2012. Microbial diversity of bovine mastitic milk as described by pyrosequencing of metagenomic $16 \mathrm{~s}$ rDNA. PLoS One, 7(10): e47671, doi: 10.1371/ journal.pone. 0047671
Orlowska A, Iwan E, Smreczak M and Rola J, 2019. Evaluation of direct metagenomics and target enriched approaches for high-throughput sequencing of field rabies viruses. J Vet Res, 63(4): 471-479, doi: 10.2478/jvetres-20190067

Palacios G, Druce J, Du L, Tran T, Birch C et al., 2008. A new arenavirus in a cluster of fatal transplantassociated diseases. N Engl J Med, 358(10): 991-998, doi: 10.1056/NEJMoa073785

Pallen MJ, 2014. Diagnostic metagenomics: potential applications to bacterial, viral and parasitic infections. Parasitology, 141(14), 1856-1862, doi: $10.1017 /$ S0031182014000134

Prakash O, Shouche Y, Jangid K and Kostka, JE, 2013. Microbial cultivation and the role of microbial resource centers in the omics era. Appl Microbiol Biotechnol, 97: 51-62, doi: 10.1007/ s00253-012-4533-y

Qiu Y, Wang S, Huang B, Zhong H, Pan Z et al., 2019. Viral infection detection using metagenomics technology in six poultry farms of eastern China. PLoS One, 14(2): e0211553, doi: 10.1371/ journal. pone. 0211553

Rampini SK, Bloemberg GV, Keller PM, Büchler AC, Dollenmaier G et al., 2011. Broad-range 16S rRNA gene polymerase chain reaction for diagnosis of culture-negative bacterial infections. Clin Infect Dis, 53(12): 1245-1251, doi: $10.1093 / \mathrm{cid} / \mathrm{cir} 692$

Rock C, Krull A, Gorden P, Shearer J and Plummer P, 2015. Metagenomic evaluation of the dairy farm environment and facilities for evidence of digital dermatitis associated bacteria. In Proceedings of the International Symposium and Conference on Lameness in RuminantsValdivia, Chile-2015

Roehe R, Dewhurst RJ, Duthie CA, Rooke JA, McKain $\mathrm{N}$ et al., 2016. Bovine host genetic variation influences rumen microbial methane production with best selection criterion for Low methane emitting and efficiently feed converting hosts based on metagenomic gene abundance. PLoS Genet, 12: e1005846, doi: 10.1371/journal.pgen. 1005846

Salipante SJ, Sengupta DJ, Rosenthal C, Costa G, 
Spangler J et al., 2013. Rapid 16S rRNA nextgeneration sequencing of polymicrobial clinical samples for diagnosis of complex bacterial infections. PloS One, 8(5): e65226, doi: 10.1371/journal.pone. 0065226

Schuenemann VJ, Singh P, Mendum TA, Krause-Kyora B, Jäger G et al., 2013. Genome-wide comparison of medieval and modern Mycobacterium leprae. Science, 341(6142): 179-183, doi: 10.1126/science. 1238286

Shabat SKB, Sasson G, Doron-Faigenboim A, Durman T, Yaacoby S et al., 2016. Specific microbiomedependent mechanisms underlie the energy harvest efficiency of ruminants. ISME J, 10(12): 2958-2972, doi: 10.1038/ismej.2016.62

Stubberfield E, AbuOun M, Sayers E, O'Connor HM and Card RM et al., 2019. Use of whole genome sequencing of commensal Escherichia coli in pigs for antimicrobial resistance surveillance, United Kingdom, 2018. Euro Surveill, 24(50):1900136, doi: 10.2807/15607917.es.2019. 24.50.1900136

Suttner B, Johnston ER, Orellana LH, Rodriguez-R LM, Hatt JK et al., 2020. Metagenomics as a public health risk assessment tool in a study of natural creek sediments influenced by agricultural and livestock runoff: potential and limitations. App Environ Microbiol, 86(6): e02525-19, doi: 10.1128/AEM.02525-19

Taylor-Brown A, Spang L, Borel N and Polkinghorne A, 2017. Culture-independent metagenomics supports discovery of uncultivable bacteria within the genus Chlamydia. Sci Rep, 7(1): 19, doi: 10.1038/s41598-017-10757-5

Towner JS, Sealy TK, Khristova ML, Albariño CG, Conlan S et al., 2008. Newly discovered Ebola virus associated with hemorrhagic fever outbreak in Uganda. PloS Pathog, 4(11): e1000212, doi: 10.1371/journal. ppat.1000212

Wagner K, Springer B, Pires VP and Keller PM, 2018. Molecular detection of fungal pathogens in clinical specimens by $18 \mathrm{~S}$ rDNA highthroughput screening in comparison to ITS PCR and culture. Sci Rep, 8: 6964, doi: 10.1038/ s41598-018-25129-w

Wallace RJ, Rooke JA, McKain N, Duthie CA, Hyslop JJ et al., 2015. The rumen microbial metagenome associated with high methane production in cattle. BMC Genomics, 16: 839, doi: 10.1186/s12864-015-2032-0

Wilson MR, Naccache SN, Samayoa E, Biagtan M, Bashir $\mathrm{H}$ et al., 2014. Actionable diagnosis of neuroleptospirosis by next-generation sequencing. N Engl J Med, 370(25): 2408-2417, doi: 10.1056/NEJMoa1401268

Xue MY, Sun HZ, Wu XH, Guan LL and Liu JX, 2019. Assessment of rumen bacteria in dairy cows with varied milk protein yield. J Dairy Sci, 102(6): 5031-5041, doi: 10.3168/jds.2018-15974

Zoetendal EG, Cheng B, Koike S and Mackie RI, 2004. Molecular microbial ecology of the gastrointestinal tract: from phylogeny to function. Curr Issues Intest Microbiol, 5: 31-48 